\title{
Comparison of blood neoangiogenesis and lymphatic vascularization in colorectal adenomas from patients with and without concomitant colorectal cancer
}

\author{
L.R. Moreira, A.A. Schenka, P. Latuf Filho, C.S.P. Lima, M.A.S. Trevisan and \\ J. Vassallo
}

Laboratório de Patologia Investigativa e Molecular, CIPED, Universidade Estadual de Campinas, Campinas, SP, Brasil

Correspondence to: J. Vassallo, Laboratório de Patologia Investigativa e Molecular, CIPED, Universidade Estadual de Campinas, Rua Tessália Viera de Camargo, 126, Caixa Postal 6111, 13083-970 Campinas, SP, Brasil

Fax: +55-19-3289-3897. E-mail: lu.patologia@uol.com.br or vassallomeister@gmail.com

Blood and lymphatic vessel proliferation is essential for tumor growth and progression. Most colorectal carcinomas develop from adenomas (adenoma-carcinoma sequence) in a process due to accumulation of molecular genetic alterations. About $5 \%$ of adenomatous polyps are expected to become malignant, but data on the differential angiogenic patterns of these lesions in patients with and without concomitant cancer are missing. The aim of the present study is to compare the angiogenic and lymphatic patterns of adenomatous polyps from patients with and without sporadic cancer. Thirty adenomatous polyps ( 15 from patients with another principal malignant lesion, and 15 from patients without cancer) were submitted to immunohistochemical staining for CD105 (marker for neoangiogenesis) and D2-40 (marker for lymphatic endothelium). Microvessel density and total vascular area were determined by computer image analysis to quantify the immunostained and total areas, and to assess the number of microvessels. Adenomas from patients with carcinoma showed significantly higher values of total vascular area determined by immunostaining for CD105 (cutoff value $=4386 \mu \mathrm{m}^{2} ; \mathrm{P}=0.019$ ) and of lymphatic microvessel density determined by immunostaining with D2-40 (cutoff value $=11.5 ; \mathrm{P}=0.041$ ) when compared with those from patients without cancer. The present data indicate a significant increase in blood microvascular area and in lymphatic microvascular counts in adenomas removed from patients with cancer.

Key words: Angiogenesis; Colorectal adenoma; Colorectal cancer; Immunohistochemistry; CD105; D2-40

Research supported by FAEPEX, Faculdade de Ciências Médicas, Universidade Estadual de Campinas, Campinas, SP, Brasil, and CNPq. Publication supported by FAPESP.

Received September 22, 2008. Accepted April 13, 2009

\section{Introduction}

Colorectal carcinoma (CRC) represents an important cause of cancer mortality in industrialized countries. Most cases $(80 \%)$ correspond to sporadic carcinomas and arise from colorectal adenomas (1). The adenoma-carcinoma sequence involves accumulations of genetic alterations causing progressive disorders in the cell cycle (2). About 5\% adenomatous polyps will probably become malignant (3).

Angiogenesis plays an important role in tumor progression and metastasis in most human solid tumors (4-7). This fact has led to new perspectives in the research of prognostic indicators and of new therapeutic strategies. The fact that $20-30 \%$ of patients with CRC treated with potentially curative surgery succumb from recurrent disease suggests that the conventional prognostic factors are not 
totally sufficient (8-11).

Most studies have evaluated angiogenesis as a potential prognostic or predictive factor in $\mathrm{CRC}$ both in early and advanced disease $(12,13)$. Comparison of literature data has been frequently hindered by variations in patient management, such as indication of adjuvant therapy, and in the methods for analysis of angiogenesis (different immunohistochemical markers, quantification methods, parameters quantified, etc.) $(12,14,15)$.

Endoglin (CD105) is a membrane glycoprotein, part of the TGF-beta receptor complex, involved in angiogenesis. Markers for this protein identify newly formed blood vessels, representing a helpful tool in the evaluation of neoangiogenesis (16). In CRC, CD105 has been correlated to prediction of metastasis (17).

$\mathrm{D} 2-40$ is a monoclonal antibody directed against the oncofetal antigen $\mathrm{M} 2 \mathrm{~A}$, present in germ cells, lymphatic endothelium, and some neoplasms such as mesotheliomas $(18,19)$. Using this antibody, it has been demonstrated that the colorectal mucosa indeed presents lymphatic vessels in normal, inflammatory and neoplastic conditions $(20,21)$. However, in contrast to CD105, present in newly formed blood vessels, the presence of D2-40 does not indicate the degree of neolymphangiogenesis. Lymphatic vessel density assessed by D2-40 has been correlated with the prediction of metastasis and with a poor outcome of CRC (22-24).

In spite of the many reports on angiogenesis in cancer, data on the differential angiogenic patterns of adenomatous lesions in patients with and without concomitant CRC are not available. The purpose of the present study was to compare blood angiogenesis and lymphatic vessels between two groups of adenomatous polyps, one from patients with concomitant CRC at another site of the mucosa, and the other from patients without carcinoma, using microvessel counting and total vascular area determination with image analysis software. Our aim was to determine potential differences between adenomatous polyps from the two groups of patients, and weather the presence of carcinoma could influence the vascularization of colorectal adenomas.

\section{Material and Methods}

\section{Tissue samples}

A retrospective study was performed on 30 low-grade adenomatous polyps removed by endoscopy or surgery from 15 patients with sporadic CRC and 15 patients without carcinoma. The latter group did not show evidence of carcinoma from the time of the procedure throughout a 5year follow-up. Hamartomatous and inflammatory polyps were excluded from the study.

The samples were selected from the files of the Department of Pathology, State University of Campinas Hospital, Campinas, SP, Brazil, and included patients diagnosed from 1987 to 2003. The group of patients with CRC consisted of 8 males and 7 females ranging from 33 to 82 years (median: 61 years); 5 cases were staged as I, 7 as II, 2 as III, and 1 as IV, according to the TNM pathological staging system (25). Only low-grade adenomatous polyps, which had been removed from the colorectal mucosa concomitantly to or soon after the diagnosis of the main malignant lesion, without the effect of neoadjuvant therapy, were included. There were 8 tubular, 6 tubulovillous, and 1 villous adenomas.

In the group of patients without a diagnosis of CRC 9 were males and 6 were females ranging from 20 to 82 years (median: 56 years). There were 11 tubular and 4 tubulovillous adenomas.

\section{Immunohistochemistry}

Tissue specimens had been fixed in $10 \%$ formalin and embedded in paraffin and 3- $\mu \mathrm{m}$ thick sections were placed on silanized slides. Endogenous peroxidase activity was quenched by incubating the slides with $3 \% \mathrm{H}_{2} \mathrm{O}_{2}$ for 10 min. Antigen retrieval was achieved by microwaving tissue sections in $10 \mathrm{mM}$ citrate buffer, $\mathrm{pH} 6.0$, in four cycles of 5 min each. Sections were incubated at room temperature for 20 min with mouse monoclonal antibodies to CD105 (Endoglin, Clone SN6h, Dako, USA; diluted 1:15) and to D2-40 (Dako, diluted 1:400). Antigen-antibody binding was detected using the Advance system (Dako). Internal and external positive and negative controls were run concomitantly in each reaction batch.

\section{Evaluation of immunohistochemistry}

Digital images from two "hot spot" fields stained by each marker were captured. One area corresponded to the upper/inner portions of the lesions, and the other to the deeper area of the polyps. The upper/inner areas were grouped together because there were some small adenomas in which both areas appeared in the same image. Digitalization was done at 200X magnification, $120 \mathrm{dpi}$, using a digital camera (Leica DFC360 FX, Leica, Germany) connected to a bright field microscope (Leica DM5000 B).

The images were examined with image analysis software (Leica QWin Standard V3, Microsystem Imaging, Leica) set to detect color intensities in a fixed and constant range. Every image was evaluated using this standardized program to quantify the proportion between stained and total areas and to assess the number of microvessels. 
Immunostained blood and lymphatic vessels were marked with a circle by the pathologist who analyzed the image to perform automated quantification. An example of the resulting image prepared for analysis after selection of the immunostained vessels is shown in Figure 1. This resulted in the evaluation of two parameters for each marker: microvessel density (MVD) and total vascular area (TVA).

\section{Statistical analysis}

Statistical analysis was performed using the SAS System for Windows software package (version 9.1.3). For the quantitative parameters, the minimum and maximum values, mean, standard deviation, and median were analyzed. For the qualitative variables, the absolute and relative frequencies were analyzed. The non-parametric MannWhitney test was used to compare two groups and the Kruskal-Wallis test was used for three or more groups. The Dunn comparison test was used for multiple comparisons. The level of significance was set at $5 \%$ in all analyses.

\section{Results}

\section{CD105 in adenomas from patients with CRC}

MVD ranged from 0 to 15 (median 5; mean 5.53) in "hot spots" of the upper/inner parts of adenomas and from 0 to 37 (median 1; mean 5.40) in the deeper areas. TVA ranged from 0 to $219905 \mu^{2}$ (median 7498; mean $25681.33 \mu \mathrm{m}^{2}$ ) in the "hot spots" of the upper/inner regions and from 0 to $27255 \mu^{2}$ (median 755; mean $6215.00 \mu \mathrm{m}^{2}$ ) in the deeper area.

\section{CD105 in adenomas from patients without CRC}

MVD ranged from 0 to 10 (median 2; mean 3.20) in "hot spots" of the upper/inner part of adenomas and from 0 to 10 (median 1; mean 2.53) in the deeper area. TVA ranged from 0 to $19660 \mu \mathrm{m}^{2}$ (median 183; mean $3810.20 \mu \mathrm{m}^{2}$ ), in the "hot spots" of the upper/inner parts and from 0 to 66522 $\mu \mathrm{m}^{2}$ (median 143; mean $8114.40 \mu \mathrm{m}^{2}$ ) in the deeper area The results for CD105 are summarized in Table 1.

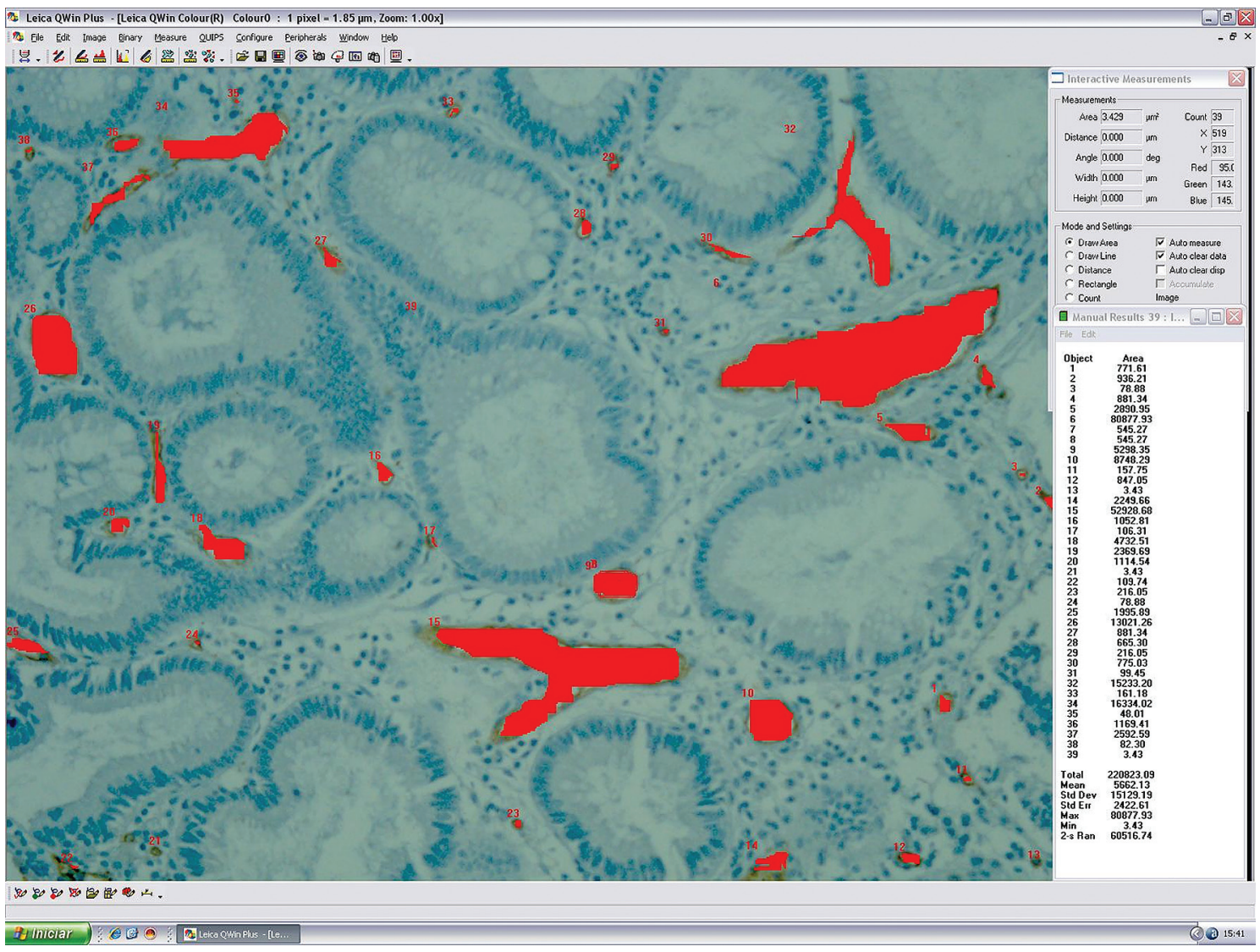

Figure 1. Evaluation of microvessels in immunostained sections by computer image analysis: the positive vessels are delimited in red and their area was calculated by software. 
Table 1. Microvessel density (MVD; number of microvessels counted) and total vascular area (TVA, $\mu \mathrm{m}^{2}$ ) determined by immunostaining for CD105 in the two areas (upper/inner and deeper) of adenomas in 15 patients with CRC and 15 patients with no CRC.

\begin{tabular}{lccccc}
\hline Variables & Area & Patient group & Range & Mean \pm SD & Median \\
\cline { 4 - 5 } MVD & Upper/inner & CRC & $0-15$ & $5.53 \pm 5.22$ & 5 \\
& & No CRC & $0-10$ & $3.20 \pm 3.41$ & 2 \\
& \multirow{4}{*}{ Deeper } & CRC & $0-37$ & $5.40 \pm 9.56$ & 1 \\
\multirow{4}{*}{ TVA } & & No CRC & $0-10$ & $2.53 \pm 3.27$ & 1 \\
& \multirow{4}{*}{ Upper/inner } & CRC & $0-219905$ & $25681.33 \pm 55338.71^{*}$ & 7498 \\
& & No CRC & $0-19660$ & $3810.20 \pm 5910.57$ & 183 \\
& \multirow{4}{*}{ Deeper } & CRC & $0-27255$ & $6215.00 \pm 9110.95$ & 755 \\
& & No CRC & $0-66522$ & $8114.40 \pm 17675.36$ & 143
\end{tabular}

$\mathrm{CRC}=$ colorectal cancer; No $\mathrm{CRC}=$ no colorectal cancer; $\mathrm{SD}=$ standard deviation. ${ }^{*} \mathrm{P}<$ 0.05 compared to No CRC (non-parametric Mann-Whitney test).

Table 2. Lymphatic microvessel density (MVD; number of lymphatic microvessels counted) and total vascular area (TVA, $\mu \mathrm{m}^{2}$ ) determined by immunostaining for D2-40 in the two areas (upper/inner and deeper) of adenomas in 15 patients with $\mathrm{CRC}$ and 15 patients with no CRC.

\begin{tabular}{lccccc}
\hline Variables & Area & Patient group & Range & Mean \pm SD & Median \\
& & & & & \\
\hline MVD & Upper/inner & CRC & $4-177$ & $29.20 \pm 44.74^{*}$ & 12 \\
& & No CRC & $1-18$ & $8.40 \pm 5.58$ & 8 \\
& \multirow{4}{*}{ Deeper } & CRC & $1-37$ & $14.67 \pm 10.31$ & 12 \\
\multirow{4}{*}{ TVA } & & No CRC & $2-14$ & $8.27 \pm 3.28$ & 8 \\
& \multirow{4}{*}{ Upper/inner } & CRC & $85-40691$ & $9232.00 \pm 11023.34$ & 3914 \\
& & No CRC & $1-53112$ & $11592.47 \pm 15384.84$ & 5836 \\
& \multirow{4}{*}{ Deeper } & CRC & $676-64650$ & $18473.73 \pm 20906.02$ & 9563 \\
& & No CRC & $265-44491$ & $14430.73 \pm 14044.96$ & 8687
\end{tabular}

$\mathrm{CRC}=$ colorectal cancer; No $\mathrm{CRC}=$ no colorectal cancer; $\mathrm{SD}=$ standard deviation. ${ }^{*} \mathrm{P}<$ 0.05 compared to No CRC (non-parametric Mann-Whitney test).

\section{D2-40 in adenomas in patients with CRC}

MVD ranged from 4 to 177 (median 12; mean 29.20) in "hot spots" of the upper/inner parts of the adenoma and TVA ranged from 85 to $40691 \mu^{2}$ (median 3914; mean $9232.00 \mu^{2}$ ). MVD ranged from 1 to 37 (median 12; mean 14.67) in "hot spots" of the deeper area, and TVA ranged from 676 to $64650 \mu \mathrm{m}^{2}$ (median 9563; mean $18473.73 \mu \mathrm{m}^{2}$ ).

\section{D2-40 in adenomas from patients without CRC}

MVD ranged from 1 to 18 (median 8; mean 8.40) in "hot spots" of the upper/inner parts of the adenoma and TVA ranged from 1 to 53112 $\mu \mathrm{m}^{2}$ (median 5836; mean 11592.47 $\mu \mathrm{m}^{2}$ ). MVD ranged from 2 to 14 (median 8; mean 8.27) in "hot spots" of the deeper area and TVA ranged from 265 to $44491 \mu \mathrm{m}^{2}$ (median 8687; mean $14430.73 \mu \mathrm{m}^{2}$ ). The results for D2-40 are summarized in Table 2. A plate with illustrations of cases with lower and higher vascularization using both markers is shown in Figure 2.

Figure 2. Various degrees of vascularization using anti-CD105 and D2-40: low and high values are shown (original magnification, 200X). A, CD105, High vascularization (arrows); $B, \mathrm{CD} 105$, low vascularization; $C$, D2-40, high vascularization (arrows); D, D2-40, low vascularization.

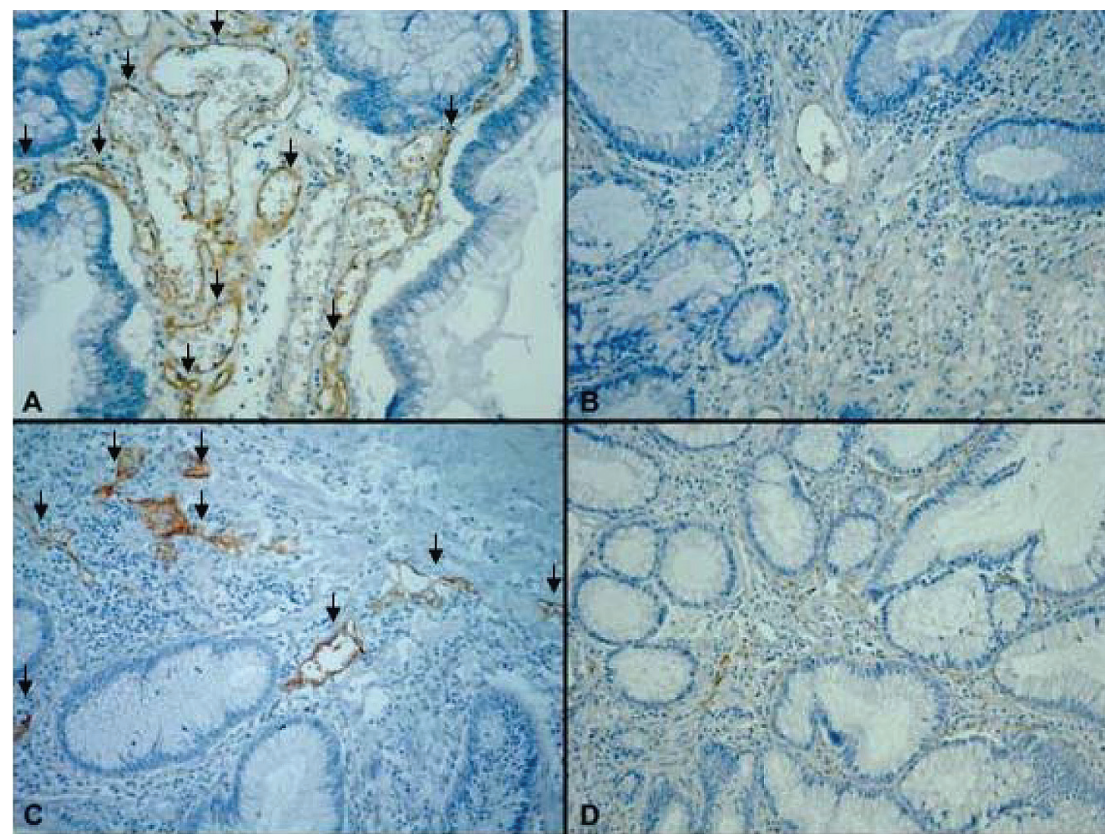


Statistical analysis of these data showed significantly higher values of TVA determined by immunostaining for CD105 $(P=0.019)$ and of MVD determined by immunostaining for $D 2-40(P=0.041)$ when compared with those from patients without $C R C$.

For both markers, there was no significant difference among histological types of adenoma (tubular, tubulovillous and villous) and MVD or TVA counts, in the groups of patients with and without CRC.

The cutoff value for TVA determined by CD105 in the upper/inner parts of the adenomas was 4386 , or approximately $4400 \mu \mathrm{m}^{2}$ (sensitivity and specificity: $66.7 \%$; predictive positive and predictive negative values: $66.7 \%$; accuracy: $66.7 \%)$. The cutoff value of MVD determined by D2-40 in the upper/inner parts of the adenomas was 11.5 (60.0\% sensitivity, $66.7 \%$ specificity, $64.3 \%$ predictive positive value, $62.5 \%$ predictive negative value, and $63.3 \%$ accuracy).

\section{Discussion}

The present data indicate a significant increase in blood microvascular area and in lymphatic microvascular counts in the upper and inner portions of adenomas removed from patients with CRC compared to those without carcinoma. Cancer cells might have an influence on the vascularization of adenomas, evidence supported by data showing increased levels of angiogenic factors in colorectal tissues distant from the primary tumor. Hanrahan et al. (26) showed that vascular endothelial growth factor (VEGF) plays a role early in tumor development at the stage of adenoma formation. Moreover, increased levels of VEGF in normal tissue collected from sites distant from the primary tumor have indicated environmental changes that could help explain our findings, although this was not directly assessed in our material.

The more significant increase in lymphatic MVD in the upper/inner areas of the adenomas is in keeping with a previous study reporting a more superficial location of lymphatic vessels in adenomas. This finding supports the hypothesis sustained by Fogt et al. (20) that superficial lymphatic vessels may be immature in normal colonic mucosa and may not communicate with deeper vessels, changing and maturing through the adenoma-carcinoma process. An equivalent assumption could be made about the increase in newly formed blood vessels detected by CD105, which suggests that they may originate superficially on adenomas, developing and meeting deeper vessels during the progression of malignancy.

The assessment of TVA using immunostaining for CD105 showed significantly higher values in adenomas from patients with CRC, while assessment of MVD did not. The opposite was seen in the assessment of lymphatic vessels using the D2-40 antibody: in contrast to MVD, TVA did not differ significantly between the two groups of lesions. These differences might reflect variations in the mechanisms of proliferation of blood and lymphatic vessels, the former affecting predominantly architectural scores, and the latter numerical scores. Unlike normal blood vessels, newly formed blood vessels incorporated during tumor angiogenesis are tortuous and dilated, a fact that could explain the higher value of TVA using CD105 in patients with CRC, an aspect supported by experimental studies (27). The higher MVD evaluated by D2-40 in patients with CRC could be explained by recent evidence showing elevated lymphatic vessel counts as an event preceding the increased number of blood vessels in early gastrointestinal tumors (28). It should be noted that computer image analysis seems to be more objective and reproducible, reducing to minimum intraobserver variability from case to case, and increasing the reliability of information in the study of angiogenesis (29).

The findings reported in the present study support the notion that neoangiogenesis and elevated lymphatic vessel counts occur in colorectal adenomas from patients with $\mathrm{CRC}$, allowing us to assume that either angiogenic factors produced by the carcinoma or constitutional defects of the colorectal epithelial cells might account for these observations.

\section{References}

1. Ilyas M, Straub J, Tomlinson IP, Bodmer WF. Genetic pathways in colorectal and other cancers. Eur J Cancer 1999; 35: 335-351.

2. Vogelstein B, Fearon ER, Hamilton SR, Kern SE, Preisinger AC, Leppert M, et al. Genetic alterations during colorectaltumor development. N Engl J Med 1988; 319: 525-532.

3. Boyle P, Leon ME. Epidemiology of colorectal cancer. $\mathrm{Br}$
Med Bull 2002; 64: 1-25.

4. Vieira SC, Zeferino LC, Da Silva BB, Aparecida PG, Vassallo $\mathrm{J}$, Carasan GA, et al. Quantification of angiogenesis in cervical cancer: a comparison among three endothelial cell markers. Gynecol Oncol 2004; 93: 121-124.

5. Offersen BV, Borre M, Sorensen FB, Overgaard J. Comparison of methods of microvascular staining and quantification 
in prostate carcinoma: relevance to prognosis. APMIS 2002; 110: 177-185.

6. Sasano H, Suzuki T. Pathological evaluation of angiogenesis in human tumor. Biomed Pharmacother 2005; 59 (Suppl 2): S334-S336.

7. Szabo S, Sandor Z. The diagnostic and prognostic value of tumor angiogenesis. Eur J Surg Suppl 1998; 99-103.

8. Bendardaf R, Lamlum H, Pyrhonen S. Prognostic and predictive molecular markers in colorectal carcinoma. Anticancer Res 2004; 24: 2519-2530.

9. Ratto C, Sofo L, Ippoliti M, Merico M, Doglietto GB, Crucitti F. Prognostic factors in colorectal cancer. Literature review for clinical application. Dis Colon Rectum 1998; 41: 10331049.

10. Buyse M, Piedbois P. Should Dukes' B patients receive adjuvant therapy? A statistical perspective. Semin Oncol 2001; 28: 20-24.

11. Reinmuth N, Parikh AA, Ahmad SA, Liu W, Stoeltzing O, Fan F, et al. Biology of angiogenesis in tumors of the gastrointestinal tract. Microsc Res Tech 2003; 60: 199-207.

12. Tarta C, da Silva V, Teixeira CR, Prolla JC, Meurer L, Neto $\mathrm{CC}$, et al. Digital image analysis and stereology of angiogenesis in polypoid and nonpolypoid colorectal adenomas. Anal Quant Cytol Histol 2004; 26: 201-206.

13. Matsuura T, Kuratate I, Teramachi K, Osaki M, Fukuda $Y$, Ito $\mathrm{H}$. Thymidine phosphorylase expression is associated with both increase of intratumoral microvessels and decrease of apoptosis in human colorectal carcinomas. Cancer Res 1999; 59: 5037-5040.

14. Compton CC. Colorectal carcinoma: diagnostic, prognostic, and molecular features. Mod Pathol 2003; 16: 376-388.

15. Pavlopoulos PM, Konstantinidou AE, Agapitos E, Kavantzas N, Nikolopoulou P, Davaris P. A morphometric study of neovascularization in colorectal carcinoma. Cancer 1998; 83: 2067-2075.

16. Thompson WD, Shiach KJ, Fraser RA, McIntosh LC, Simpson JG. Tumours acquire their vasculature by vessel incorporation, not vessel ingrowth. J Pathol 1987; 151: 323-332.

17. Romani AA, Borghetti AF, Del Rio P, Sianesi M, Soliani P. The risk of developing metastatic disease in colorectal cancer is related to CD105-positive vessel count. J Surg Oncol 2006; 93: 446-455.

18. Van den Eynden GG, Van der Auwera I, Van Laere SJ, Colpaert CG, van Dam P, Dirix LY, et al. Distinguishing blood and lymph vessel invasion in breast cancer: a prospective immunohistochemical study. $\mathrm{Br} J$ Cancer 2006;
94: 1643-1649.

19. Ordonez NG. Podoplanin: a novel diagnostic immunohistochemical marker. Adv Anat Pathol 2006; 13: 83-88.

20. Fogt F, Zimmerman RL, Ross HM, Daly T, Gausas RE. Identification of lymphatic vessels in malignant, adenomatous and normal colonic mucosa using the novel immunostain D2-40. Oncol Rep 2004; 11: 47-50.

21. Fogt F, Pascha TL, Zhang PJ, Gausas RE, Rahemtulla A, Zimmerman RL. Proliferation of D2-40-expressing intestinal lymphatic vessels in the lamina propria in inflammatory bowel disease. Int J Mol Med 2004; 13: 211-214.

22. Longatto-Filho A, Pinheiro C, Ferreira L, Scapulatempo C, Alves VA, Baltazar F, et al. Peritumoural, but not intratumoural, lymphatic vessel density and invasion correlate with colorectal carcinoma poor-outcome markers. Virchows Arch 2008; 452: 133-138.

23. Yan G, Zhou XY, Cai SJ, Zhang GH, Peng JJ, Du X. Lymphangiogenic and angiogenic microvessel density in human primary sporadic colorectal carcinoma. World J Gastroenterol 2008; 14: 101-107.

24. Matsumoto $K$, Nakayama $Y$, Inoue $Y$, Minagawa N, Katsuki $\mathrm{T}$, Shibao K, et al. Lymphatic microvessel density is an independent prognostic factor in colorectal cancer. Dis CoIon Rectum 2007; 50: 308-314.

25. International Union against Cancer. TNM classification of malignant tumours. 5th edn. Geneva: International Union against Cancer (http://www.uicc.org); 2004.

26. Hanrahan V, Currie MJ, Gunningham SP, Morrin HR, Scott $\mathrm{PA}$, Robinson $\mathrm{BA}$, et al. The angiogenic switch for vascular endothelial growth factor (VEGF)-A, VEGF-B, VEGF-C, and VEGF-D in the adenoma-carcinoma sequence during colorectal cancer progression. J Pathol 2003; 200: 183-194.

27. Vermeulen PB, Gasparini G, Fox SB, Colpaert C, Marson $L P$, Gion M, et al. Second international consensus on the methodology and criteria of evaluation of angiogenesis quantification in solid human tumours. Eur J Cancer 2002; 38: 1564-1579.

28. Gao Y, Zhong WX, Mu DB, Yuan YP, Zhang YH, Yu JM, et al. Distributions of angiogenesis and lymphangiogenesis in gastrointestinal intramucosal tumors. Ann Surg Oncol 2008; 15: 1117-1123.

29. Van der Auwera I, Cao Y, Tille JC, Pepper MS, Jackson DG, Fox SB, et al. First international consensus on the methodology of lymphangiogenesis quantification in solid human tumours. Br J Cancer 2006; 95: 1611-1625. 